\title{
Microstructural Comparison of La-V-O Compounds prepared by Sol-Gel Acrylamide Polymerization and Solid State Reaction.
}

\author{
G. Herrera*, E. Chavira $^{* *}$, J. Jiménez-Mier*, A. Ordoñez ${ }^{\dagger}$, E. Bucio ${ }^{*}$ L. Baños ${ }^{* *}$, J. \\ Guzmán $^{* * *}$, E. Fregoso-Israel ${ }^{* *}$ O. Novelo ${ }^{* *}$, \\ *Instituto de Ciencias Nucleares. Universidad Nacional Autónoma de México, 70-543, 04510 \\ México D. F., MEXICO. guillermo.herrera@nucleares.unam.mx \\ ** Instituto de Investigaciones en Materiales. Universidad Nacional Autónoma de México, 70- \\ 360,04510 México D. F., MEXICO.
}

$\mathrm{LaVO}_{4}$ and $\mathrm{LaVO}_{3}$ are lanthanum orthovanadates that belong to a group of compounds with interesting structural, electronic, magnetic and electrical properties. The crystal structure of $\mathrm{LaVO}_{4}$ compound has been reported in two polymorphs, namely, tetragonal zircon-type ( $t$ $\left.\mathrm{LaVO}_{4}\right)$ isostructural to $\mathrm{ZrSiO}_{4}$ compound and monoclinic monazite-type $\left(\mathrm{m}-\mathrm{LaVO}_{4}\right) . \mathrm{LaVO}_{3}$ compound has an orthorhombic distorted perovskite structure first found in $\mathrm{GdFeO}_{3}$ compound. The purpose of this work is to compare the influence of sol-gel acrylamide polymerization synthesis on the crystal structure and microstructure in $\mathrm{m}-\mathrm{LaVO}_{4}$ and $\mathrm{LaVO}_{3}$. These results were contrasted with the samples obtained by solid state reaction (SSR). The differential thermal analysis (DTA) for SGAP shows the formation of $m-\mathrm{LaVO}_{4}$ occurs at $400{ }^{\circ} \mathrm{C}$, in comparison with the sample prepared by SSR at $1400{ }^{\circ} C$ [1].

Fig. 1 (a) shows the morphology and roughness obtained by atomic force microscopy (AFM). The image reveals needle shape particles (needles are made of metal-EDTA and polymer). This crystallization style depends of EDTA molar concentration and $\mathrm{pH}$ value. We use a solution of 1:1 vanadium-EDTA molar ratio and we adjusted the $\mathrm{pH}$ to 5.4 with $\mathrm{NH}_{4} \mathrm{OH}$. Because the fast acrylamide polymerization is generally in aqueous medium whose $\mathrm{pH}$ is close to neutral [2]. It has been reported that weak ligand such as EDTA adjusts the morphology and uniformity of crystals shape in the crystallization process. Also, Jia et al. [3] reported that the $\mathrm{pH}$ value ranged from 7-13 exhibited rods like morphology. The needle shape was maintained up to the formation of $\mathrm{LaVO}_{4}$ compound. This result was confirmed by scanning electron microscopy (SEM) micrographs, see Fig. 1 (b). The $m$ - $\mathrm{LaVO}_{4}$-SGAP reveals a homogeneous morphology with needle-shaped grains of $50 \mathrm{~nm}$ average size. The SSR present a broader size distribution in the micrometer range.

Both $\mathrm{m}$ - $\mathrm{LaVO}_{4}$ samples were reduced into $\mathrm{LaVO}_{3}$ using a $\mathrm{Zr}$ rod at $850{ }^{\circ} \mathrm{C}$ in vacuum. Fig. 2 (right) shows a homogeneous grain distribution with an average size of $745 \mathrm{~nm}$ for $\mathrm{LaVO}_{3^{-}}$ SGAP. $\mathrm{LaVO}_{3}$-SSR has an average size of $3.45 \mu \mathrm{m}$ (Fig. 2, left). The stoichiometry of all compounds was confirmed by energy dispersive X-ray spectroscopy (EDX). X-ray powder diffraction (XRD) and transmission electron microscopy (TEM) give crystal structures in agreement with those reported in the literature. An image from TEM study for $\mathrm{LaVO}_{3}-\mathrm{SGAP}$ is shown in Fig. 3. The morphology is in agreement with SEM results.

References

[1] G. Herrera et al., J. Alloys Compd. (2009) in press.

[2] A. Sin and P. Odier. Adv. Mater. 12, 9 (2000) 649.

[3] C. Jia, et al., J. Phys. Chem. B 109 (2005) 3284.

[4] Research supported by grants UNAM-PAPIIT IN109308; CONACYT U41007-F and 56764. 

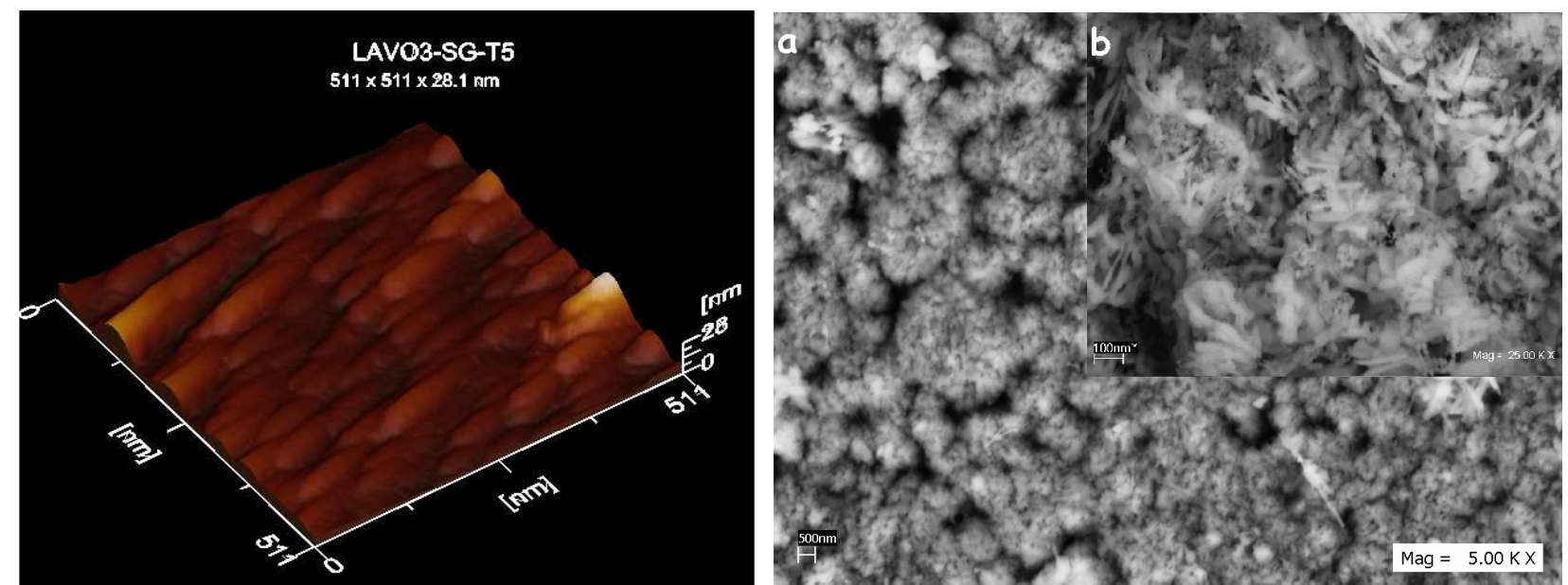

Fig. 1 (a) AFM micrograph obtained by AC mode of gel prepared by SGAP. The image size is $511 \times 511 \mathrm{~nm}$ and clock speed of $666.70 \mu \mathrm{s}$. (b) SEM micrograph of $\mathrm{m}$ - $\mathrm{LaVO}_{4}$-SGPA at the end of synthesis at $400{ }^{\circ} \mathrm{C}$ during 12 hours in air.
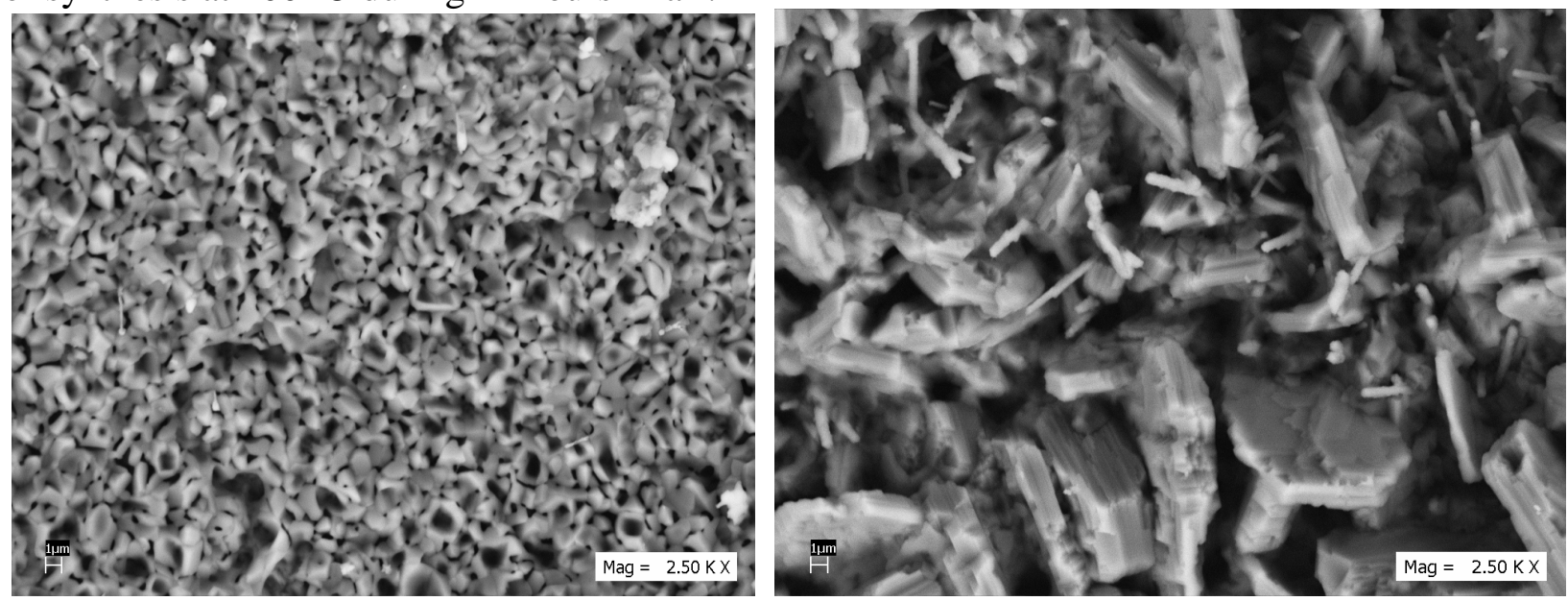

Fig. 2. Comparison of microstructure obtained by SEM on surface of $\mathrm{LaVO}_{3}$ compound at the end of heat treatment at $850{ }^{\circ} \mathrm{C}$ during 15 days prepared by SGAP (right). SEM micrograph of surface of $\mathrm{LaVO}_{3}$ compound into a pellet topology at $850{ }^{\circ} \mathrm{C}$ during 15 days prepared by SSR.
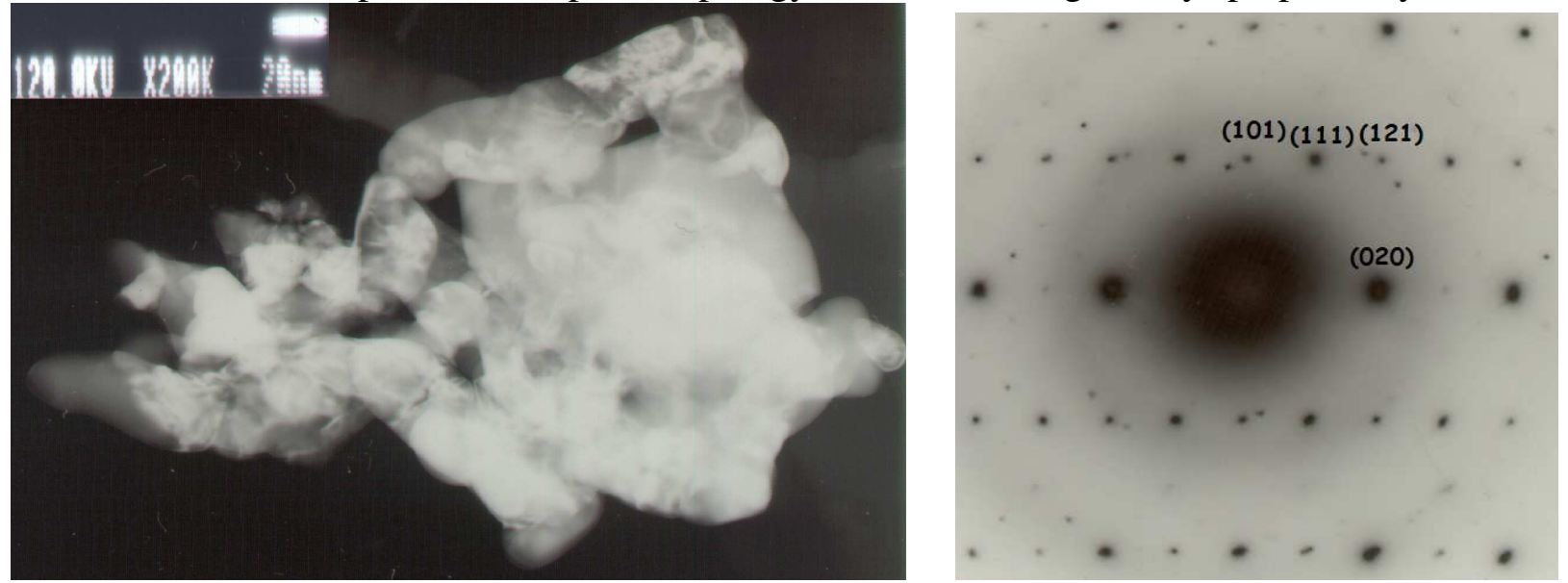

Fig. 3. TEM micrograph powders of $\mathrm{LaVO}_{3}$ compound prepared by SGAP and indexed diffraction pattern of [101] zone axe. 